\title{
BMJ Open Quality Improving annual albuminuria testing for individuals with diabetes
}

To cite: Kam S, Angaramo S, Antoun J, et al. Improving annual albuminuria testing for individuals with diabetes. BMJ Open Quality 2022;11:e001591. doi:10.1136/ bmjoq-2021-001591

Received 23 June 2021 Accepted 20 January 2022

Check for updates

(c) Author(s) (or their employer(s)) 2022. Re-use permitted under CC BY-NC. No commercial re-use. See rights and permissions. Published by BMJ.

${ }^{1}$ Vanderbilt University School of Medicine, Nashville, Tennessee, USA

${ }^{2}$ Department of Medicine,

Division of Diabetes,

Endocrinology, and Metabolism, Vanderbilt University Medical Center, Nashville, Tennessee, USA

Correspondence to Dr Chase D Hendrickson; chase.d.hendrickson@ vanderbilt.edu

\section{ABSTRACT}

Background Annual albuminuria screening detects the early stages of nephropathy in individuals with diabetes. Because early detection of albuminuria allows for interventions that lower the risk of developing chronic kidney disease, guidelines recommend annual testing for all individuals with type 2 diabetes mellitus and for those with type 1 diabetes for at least 5 years. However, at the Eskind Diabetes Clinic at the Vanderbilt University Medical Center, testing occurred less frequently than desired. Methods A quality improvement team first analysed the clinic's processes, identifying the lack of a systematic approach to testing as the likely cause for the low rate. The team then implemented two successive interventions in a pilot of patients seen by nurse practitioners in the clinic. In the first intervention, staff used a dashboard within the electronic health record while triaging each patient, pending an albuminuria order if testing had not been done within the past year. In the second intervention, clinic leadership sent daily reminders to the triage staff. A statistical process control chart tracked monthly testing rates.

Results After 6 months, annual albuminuria testing increased from a baseline of $69 \%$ to $82 \%$, with multiple special-cause signals in the control chart.

Conclusions This project demonstrates that a series of simple interventions can significantly impact annual albuminuria testing. This project's success likely hinged on using an existing workflow to systematically determine if a patient was due for testing and prompting the provider to sign a pended order for an albuminuria test. Other diabetes/endocrinology and primary care clinics can likely implement a similar process and so improve testing rates in other settings. When coupled with appropriate interventions to reduce the development of chronic kidney disease, such interventions would improve patient outcomes, in addition to better adhering to an established quality metric.

\section{INTRODUCTION}

\section{Background}

Diabetic kidney disease (DKD), also known as diabetic nephropathy, ranks as the leading cause of end-stage renal disease (ESRD) in the USA and is an independent risk factor for the development of cardiovascular disease. ${ }^{12}$
DKD occurs in $20 \%-40 \%$ of individuals with diabetes, typically developing about 10 years after a diagnosis of type 1 diabetes mellitus (T1DM) and any time after a diagnosis of type 2 diabetes mellitus (T2DM). ${ }^{1}$ In the USA, diabetes mellitus (DM) affects about $10.5 \%$ of the population ( 34.2 million people), and DKD affects about $38.6 \%$ of those diagnosed ( 288 000 people) ${ }^{3}$ For people with diabetes with chronic kidney disease (CKD) stages 2, 3 or 4 , the risk of progression to dialysis over a 5 -year period is $1 \%, 1 \%$ and $20 \%$, respectively, with corresponding mortality rates of $20 \%, 24 \%$ and $46 \% .{ }^{4}$ DKD significantly impacts financial spending, with Medicare expenditures for CKD and ESRD exceeding $\$ 120$ billion in 2017. ${ }^{2}$

The pathogenesis of DKD is complex and multifactorial. Many modifiable and nonmodifiable risk factors lead to the development of $\mathrm{DKD}^{4}$ Non-modifiable factors include advanced age, age of diabetes onset, genetic factors and family history. Modifiable factors include poor glycaemic control, hypertension, dyslipidaemia, obesity, smoking and socioeconomic disadvantage. Overall, DKD is understood to be a consequence of hyperglycaemia causing microvascular damage to the renal glomerulus milieu. ${ }^{45}$ This pathological process initially manifests as glomerular hyperfiltration, followed by worsening renal function and eventually hypofiltration. ${ }^{6}$ Albuminuria signals glomerular hyperfiltration in the early stages.

The American Diabetes Association (ADA) recommends annual albuminuria testing for all patients with T2DM and for patients with T1DM for $\geq 5$ years. ${ }^{1}$ Common testing tools include a spot urine albumin-to-creatinine ratio (UACR) or a 24-hour creatinine collection. $^{7}$ The UACR is recommended and preferred due to the ease of test timing and a relatively inexpensive cost. 
With appropriate intervention, early detection of albuminuria leads to medical interventions that prevent or slow progression of DKD. ${ }^{57}$ Optimising glucose control decreases onset and worsening nephropathy by $33 \% .{ }^{8}$ The addition of an ACE inhibitor (ACE-I), angiotensinreceptor blocker (ARB) or other diuretic helps prevent or slows the development of nephropathy, ${ }^{9}{ }^{10}$ as does the addition of a sodium-glucose cotransporter 2 inhibitor $^{11} 12$ or a glucagon-like peptide-1 agonist. ${ }^{13}$ Models suggest that annual albuminuria testing of individuals with diabetes is a cost-effective strategy. ${ }^{1915}$

\section{Available knowledge}

Past quality improvement (QI) projects report a wide range of strategies in DM management to improve the frequency of albuminuria testing. These include implementing a diabetes education programme for providers and patients, ${ }^{16}$ providing a decision support tool in the form of a paper flow chart ${ }^{17}$ and creating a programme using patient navigators. ${ }^{18}$ Some studies in primary care settings used clinical support tools and provider education to improve testing rate disparities related to patient minority status ${ }^{19-21}$ or uninsured status. ${ }^{22}$

Several QI studies focused on electronic health record (EHR)-based interventions. These EHR-based reminders and monitoring improved compliance with albuminuria testing in primary care settings. ${ }^{23}{ }^{24}$ The development of EHR templates and order sets increased adherence to testing guidelines in a paediatric endocrine clinic $^{25}$ and in a primary care setting. ${ }^{26}$

\section{Problem description, rationale and global aim}

The Eskind Diabetes Clinic (EDC) at the Vanderbilt University Medical Center (VUMC) employs no systematic process to ensure that albuminuria testing is up to date for patients with DM. While the triage team assists clinicians with addressing other diabetes maintenance testing parameters (haemoglobin A1C, diabetic foot exam and retinopathy testing), no similar workflow addresses albuminuria testing. Testing depends solely on provider recall during patient visits, and some clinicians have developed personalised standard visit checklists to minimise missed screens.

EDC clinicians raised awareness of the lack of a systembased approach and the potential for a negative impact on patients by not consistently following this standard of care. ${ }^{1}$ They indicated that the rate of annual albuminuria testing was likely to be lower than desired for this reason. We hypothesised that substandard testing likely existed and that introducing a systematic workflow could alleviate the clinician burden of relying on memory and so improve albuminuria testing rates. Thus, we created a project with the global aim to increase the frequency with which patients in the EDC received annual albuminuria testing.

\section{METHODS}

Setting

VUMC is in Nashville, Tennessee, and manages over 2 million patients yearly from Tennessee and neighbouring

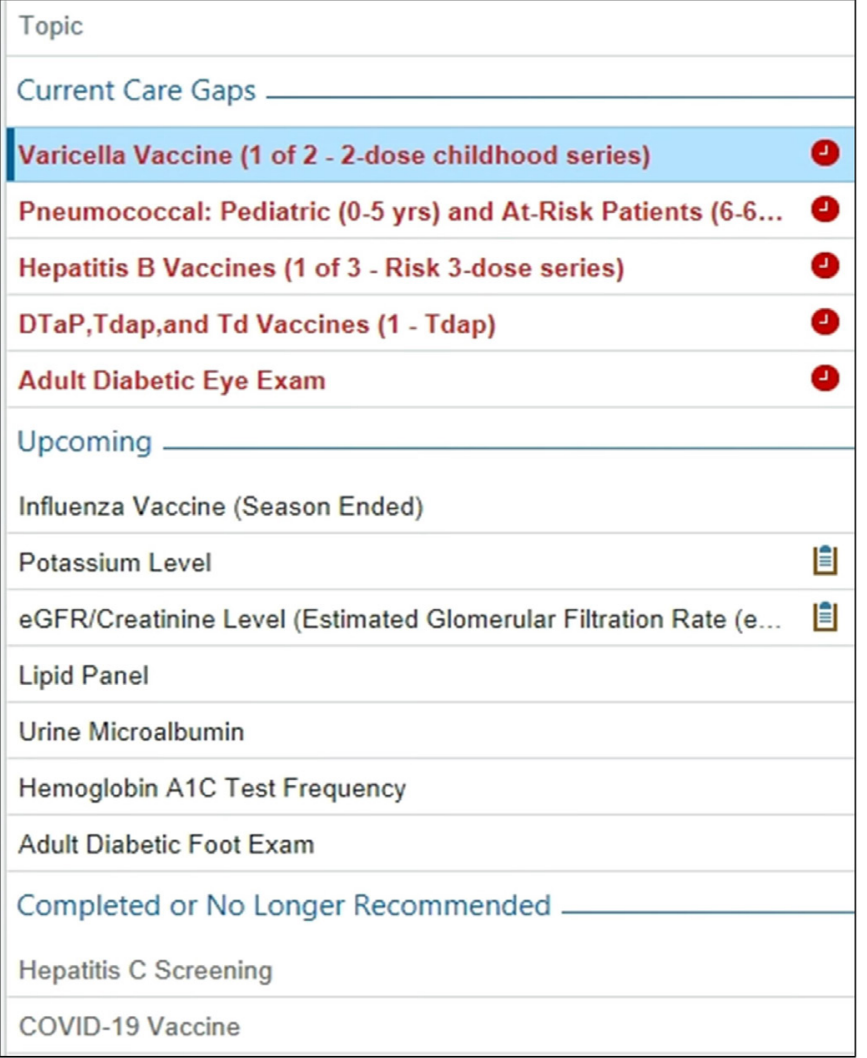

Figure 1 Example of a patient dashboard as viewed by triage staff for a patient with diabetes. 2021 Epic Systems Corporation.

states. While located in an urban setting, VUMC serves patients representing a wide range of socioeconomic and cultural backgrounds. VUMC uses a single EHR (Epic Systems, Verona, Wisconsin, USA) integrated across all inpatient and outpatient sites, including laboratory and radiology services, that has been in place since November 2017.

The EDC is a multidisciplinary outpatient endocrinology clinic within VUMC, serving 9500 individuals with DM annually. Physicians and nurse practitioners (NPs) provide team-based care for these patients, with patients seeing a physician at least annually and NPs at different intervals as needed. During in-person visits, medical assistants (MAs) and licenced practical nurses (LPNs) triage patients, including documenting the date of the most recent diabetic eye and foot exams and performing pointof-care haemoglobin A1C testing when indicated. Triage staff use a dashboard within the EHR (see figure 1 for an example) to determine and document what testing is due for each patient. They manually record dates for the most recent foot and eye exams, and dates of the most recent $\mathrm{AlC}$ and albuminuria tests import automatically from the VUMC lab database. The triage process ends with escorting patients to the clinic room for the clinician visit. After visits are complete, patients check-out at the front desk and are directed to the in-house laboratory if the clinician signed lab orders prior to checking out. 


\section{Context, design and patient involvement}

Rotating teams of three to five senior medical students at the Vanderbilt School of Medicine conducted this QI project during a 1 month diabetes elective offered during the 2020-2021 academic year. A VUMC clinician mentor trained in QI methodology provided longitudinal supervision. Each month's team completed a unique step in the project's plan-do-study-act (PDSA) cycles, including baseline analysis, stakeholder/patient engagement, development of aims and metrics, designing interventions, data analysis and dissemination of results. Each team prepared written and video hand-offs to orient the next team. Hand-offs included details on the project overall, the work completed that month and the work to be completed the next month.

The project began in August 2020 with an internal review process of albuminuria testing at the EDC. The team consulted clinicians, clinic and laboratory staff and administrators to understand the baseline processes. The team interviewed several patients after routine visits in the clinic to better understand their perceptions of why testing was being performed and barriers to successfully completing testing, with subsequent interventions designed to account for identified issues. The internal review process focused on in-person visits. The clinic operated with a hybrid model of in-person and telemedicine visits due to the COVID-19 pandemic. VUMC's telemedicine workflows regularly changed in response to the evolving situation, leading to the decision to exclude telemedicine workflows from the initial analysis and interventions. About $50 \%$ of patient visits occurred in-person at the beginning of the project and increased to $70 \%$ at the end.

Several consistent themes emerged for causes of lowerthan-target albuminuria testing rates. Provider factors included not understanding testing guidelines, omitting an albuminuria screen review or forgetting to place an EHR order. Patient factors included not stopping at check-out to be directed to the laboratory, not having time to submit a urine sample, not being able to submit a urine sample or declining to provide a urine sample because the reason for the test was not explained prior to them checking out. Figure 2 depicts the baseline process as a flow map, with the identified causes for system failure highlighted.

Following the review process, the team proposed several potential intervention ideas. These included: (A) providing patient education on the purpose of annual albuminuria testing; (B) sending an EHR-based previsit notification of an upcoming urine screen; (C) placing annual testing reminders in the clinic waiting room; (D) providing a urine sample cup to every patient at check-in; (E) introducing a review of the patient's last urine screen date during the triage check-in process; or $(\mathrm{F})$ providing additional clinician education.

We hypothesised that standardised, system-level, EHRbased interventions would be the most effective for increasing albuminuria testing rates. Based on the flow of

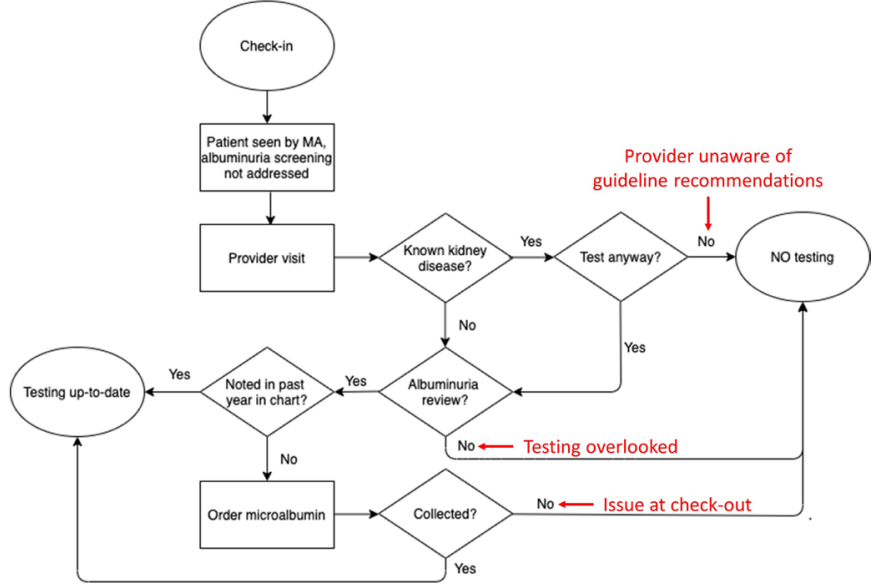

Figure 2 Flow chart of an in-clinic visit for a patient with diabetes, focused on albuminuria testing, with potential causes for system failures highlighted by arrows. MA, medical assistant.

the clinic and logistics of each proposed intervention, we aimed to focus on the triage process for our initial interventions. The triage workflow already included accessing the patient's dashboard to determine if A1C testing was due, and reviewing the status of albuminuria testing could be incorporated in the same step. Furthermore, MA/LPNs have the ability to pend orders for clinicians to sign at the conclusion of each patient visit, potentially reminding providers that albuminuria testing was due without providers needing to make this determination themselves. The triage staff indicated that such an additional step was likely to add little additional time and effort to their existing process.

The team piloted this project among the five NPs in the EDC who conducted in-person visits for patients with DM throughout the study period. The team solicited the NPs' engagement for this pilot study given their high volume of DM patient visits and interest in participating in innovative, system-based approaches to improving care.

\section{Measures}

The number and proportion of overdue versus up-todate albuminuria screens were abstracted from the EHR central database for every calendar month. Each patient visit represented a unique data point, as each visit represents an opportunity for 'success' or 'failure' of the system to appropriately test patients. Patients with multiple visits during the study period had multiple unique data points recorded in our dataset. Up-to-date albuminuria screens were defined as a spot UACR test completed on the day of or during the 364 days prior to the visit. Only tests completed within the VUMC network were included, as data from outside laboratories are not integrated into the VUMC lab record. All patient visits using an International Classification of Diseases, Tenth Revision (ICD-10) code of E8-13 were included, representing T1DM, T2DM and other specified forms of diabetes, regardless of duration of diabetes. Exclusion criteria included patients with gestational diabetes, ESRD on dialysis or those with a history 
of kidney transplantation. We did not exclude patients on ACE-I or ARB therapy or with a previous diagnosis of albuminuria, as the ADA recommends annual testing for these patients. ${ }^{1}$

\section{Specific aims}

We aimed to improve the frequency of albuminuria testing by $20 \%$ (absolute from the baseline) over a 6 -month period. We chose a relatively modest goal due to the time constraints of the project.

\section{Statistical analysis}

Data were deidentified and analysed using a p-chart, with standard rules for assessing for a special cause signal and thus statistical significance.

\section{Interventions}

PDSA cycle 1

Starting in December 2020, the team asked the triage staff to begin: (1) determining whether patients with DM were due for albuminuria testing based on a review of the EHR dashboard and (2) pending orders for an albuminuria test if one had not been performed at a VUMC facility within the past year.

For clinicians, the pended order became visible immediately when opening the patient's chart. The clinician could sign, delete or defer the order at their discretion. The EHR requires that pended orders be addressed prior to signing the visit, which typically occurs shortly after the patient leaves the clinic room.

\section{PDSA cycle 2}

During the review of PDSA cycle 1, the MAs and LPNs raised concerns regarding having difficulty remembering when to implement the intervention workflow, given the pilot's focus on only NPs at the EDC and each MA/LPN having different provider assignments daily. Thus, PDSA cycle 2 focused on creating a reminder system to improve rates of intervention implementation.

In January 2021, a reminder statement was added to the provider assignment email that the triage team received each morning. This statement was brief (a single sentence long) and was subsequently standardised for all future emails.

\section{RESULTS}

\section{Baseline data}

At the EDC, annual albuminuria testing for patients with DM averaged $69.0 \%$ of 2860 visits in the 12 months prior to this project, with the analysis indicating a stable process (see figure 3). Two months (April and May 2020) were excluded from the analysis, given the low number of in-person visits because of the COVID-19 pandemic.

\section{Postintervention data}

The postintervention period encompassed 6 months (December 2020-May 2021) and included 1245 visits. A special cause signal (monthly average above the upper
A
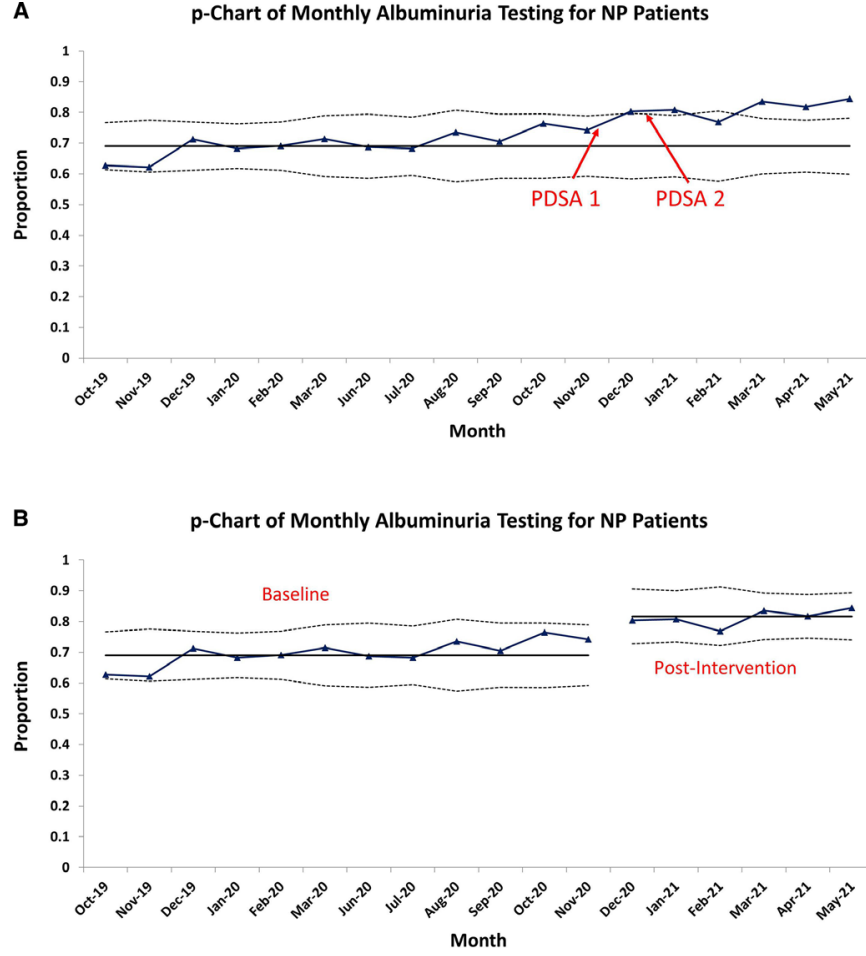

Figure 3 Statistical process control charts of the proportion of patients seen by a nurse practitioner (NP) in the clinic, with (A) a fixed mean based on the baseline performance and (B) two separate means based on preintervention and postintervention data.

control limit) occurred the first month (see figure 3A). For these 6 months, five monthly averages were above the upper control limit, with all six being well above the baseline average. Splitting the p-chart into separate preintervention and postintervention periods showed a postintervention annual albuminuria testing average of $81.7 \%$, a $12.7 \%$ increase above the baseline (see figure 3B). The special cause signals indicated that this improvement met statistical significance, though it failed to meet the prespecified goal of a $20 \%$ improvement.

\section{DISCUSSION}

\section{Summary}

Over the course of 6 months, two simple interventions increased the frequency of annual albuminuria testing from $69 \%$ to $82 \%$ at the EDC. With the first intervention, triage staff identified patients due for testing and pended an order when needed. With the second intervention, clinic leadership used an existing daily communication to remind triage staff of this process.

\section{Sustainability, Strengths, and Interpretation}

This QI project demonstrated how small, scalable, generalisable modifications to our triage process significantly improved albuminuria testing rates. To better understand the potential for negative impacts of these interventions (eg, a lengthier triage process), the team interviewed triage staff, clinic leadership and clinic providers at the conclusion of the project. All stated 
that the impact of these interventions on their work was negligible, suggesting that this work can be sustained. No one reported concerns for standardised implementation clinic wide.

Our most significant change involved standardised utilisation of our EHR's dashboard. Our dashboard is designed to be easily visible within each patient's chart and includes both diabetes parameters and non-diabetes parameters, with the diabetes parameters clustered together to allow simultaneous review. EHR dashboards are increasing in popularity as they have shown to improve patient care. ${ }^{27-31}$ Most likely, the ease of accessibility of the dashboard used in our EHR contributed to the success in the triage staff using it and would likely need to be a component for a similar intervention elsewhere to be successful.

Our secondary change focused on a reminder system for the triage teams. Based on the EDC's workflow, a slight modification to the template of the daily assignment email allowed an existing process to be adapted for this intervention. We theorise that such a reminder will not be needed after this intervention becomes routine in our clinic, and it may not have been required at all if the project was not initially piloted on only a subset of the patient encounters.

In comparison with other EHR-based QI interventions described in the literature, our interventions allow individualised review of patient testing status without excess burden on the clinical team. Anabtawi and Mathew created a computer-generated reminder that appeared every time a patient chart is opened within their primary care clinic network. ${ }^{23}$ The impact of their intervention compares well to ours, increasing test ordering from $56 \%$ to $70 \% .{ }^{23}$ While EHR-based reminder systems or clinician decision support tools are thorough, frequent alerts affect clinician focus and lead to alert fatigue. ${ }^{32} 33$ Deem $e t a l^{24}$ added the CPT code for albuminuria testing to a user-friendly short list for their primary care clinic's EHR, improving testing from $8 \%$ to $15 \%$. While similar to our use of a dashboard, this intervention did not assist clinicians with determining appropriateness of an albuminuria test. Choudhary et $a l^{25}$ used EHR templates and order sets developed for their urban communitybased paediatric endocrine clinic. While their improvement was of a greater magnitude over a 2-year period $(49 \%-79 \%)$, the volume at their site was much lower than ours and so is perhaps not comparable. Finally, Herrin $e t a l^{26}$ developed a package tool that included a 'Diabetes Self-Education' tab for passive clinical decision support and documentation tool, which was available to an ambulatory medical practice group with 156 primary and specialty care centres. Their study improved testing by $9 \%$ relative to a non-intervention group. Our triage team check is a process change most similar to these interventions but is more efficiently customised to each patient: the pended order indicates that the albuminuria testing review already occurred and that testing is due. If placing a standardised order set, the clinician still needs to perform an albuminuria testing review, which may be less efficient.

Overall, our QI interventions can be easily expanded to other diabetes clinics and primary care settings, improving the quality of diabetes care by better conforming care to accepted standards. ${ }^{1}$ By better identifying the onset and progression of albuminuria, interventions proven to prevent or slow DKD can be started more frequently, ${ }^{1}$ potentially lowering the frequency of ESRD.

\section{Limitations}

This pilot study involved a limited number of our patients, as we focused on visits with certain providers. However, our interventions can be easily expanded to the rest of our patient panel and may in fact be more successful because our triage team would standardise the intervention for all patients instead of remembering which providers were participating in the pilot study. Additionally, due to the structure of the year-long course in which the medical students participated and the need to contain the project within this time, we implemented just two PDSA cycles, with 6 months of postintervention data. While these changes have been implemented into the permanent workflow of the clinic, the effects will need to be monitored to demonstrate sustainability.

Importantly, this project required buy-in from our triage team. Certain MA/LPNs may have adapted to the new workflow more easily, which potentially influenced testing compliance rates. We were unable to track albuminuria testing rates by individual MA/LPN and so are uncertain as to presence of variable individual performance.

Our triage team has both access to the EHR's dashboard and the ability to initiate orders to be later signed by the provider. Variable policies on accessing the EHR and initiating orders in different healthcare settings could impact the ability to implement such an intervention elsewhere.

For patients who receive care outside of VUMC, some testing was likely duplicative. Outside lab reports are stored as scanned copies and do not automatically populate into the EHR, so they are easily overlooked even when obtained from primary care providers or nephrologists outside of VUMC. Given the importance of obtaining this testing annually, both to ensure good patient care and to document compliance to payers, the decision was made to accept the inevitability of some degree of duplication. Given the nature of the problem, the degree of duplication is unknown. Future efforts to integrate laboratory result systems could alleviate resource overutilisation. ${ }^{34}$

\section{CONCLUSIONS}

This report describes an effective QI intervention for increasing albuminuria testing rates that can be easily applied and scaled at other clinics with integrated EHR systems. Our intervention occurs during the patient triage process and results in minimal interruption to current workflows. The implementation capitalises on existing processes and is likely generalisable to other clinics. 
Improvements in albuminuria testing should have longstanding effects on DKD outcomes in patients with DM.

Acknowledgements We thank Amanda Carter for her leadership in the implementation of this project and Brannan Cole, Norma Edwards, Regina Hamlet, Tiffanie Marksbury, and Jennifer Prager for their participation in this work.

Contributors All listed authors contributed to the improvement work and revision of the manuscript. Additionally, SK prepared the initial drafts of the manuscript, and MJF and CDH supervised the work. All other authors share the role of second author. $\mathrm{CDH}$ serves as guarantor, accepting full responsibility for the finished work and the conduct of the study, having had access to the data and controlling the decision to publish.

Funding The authors have not declared a specific grant for this research from any funding agency in the public, commercial or not-for-profit sectors.

Competing interests None declared.

Patient and public involvement Patients and/or the public were involved in the design, or conduct, or reporting, or dissemination plans of this research. Refer to the Methods section for further details.

Patient consent for publication Not required.

Ethics approval The study protocol was reviewed and granted exemption by the Vanderbilt Institutional Review Board (IRB \#202224).

Provenance and peer review Not commissioned; externally peer reviewed.

Data availability statement Data are available upon request.

Open access This is an open access article distributed in accordance with the Creative Commons Attribution Non Commercial (CC BY-NC 4.0) license, which permits others to distribute, remix, adapt, build upon this work non-commercially, and license their derivative works on different terms, provided the original work is properly cited, appropriate credit is given, any changes made indicated, and the use is non-commercial. See: http://creativecommons.org/licenses/by-nc/4.0/.

ORCID iD

Adrian G Cadar http://orcid.org/0000-0002-1221-0764

\section{REFERENCES}

1 American Diabetes Association. Microvascular complications and foot care. Diabetes Care 2021;44:S151-67.

2 Saran R, Robinson B, Abbott KC, et al. Us renal data system 2019 annual data report: epidemiology of kidney disease in the United States. Am J Kidney Dis 2020;75:A6-7.

3 Centers for Disease Control and Prevention. National diabetes statistics report, 2020. Atlanta, GA: centers for disease control and prevention, US. Dept of Health and Human Services, 2020.

4 Thomas MC, Brownlee M, Susztak K, et al. Diabetic kidney disease. Nat Rev Dis Primers 2015;1:15018.

5 León Jiménez D, Cherney DZI, Bjornstad P, et al. Antihyperglycemic agents as novel natriuretic therapies in diabetic kidney disease. Am J Physiol Renal Physiol 2018;315:F1406-15.

6 Tonneijck L, Muskiet MHA, Smits MM, et al. Glomerular hyperfiltration in diabetes: mechanisms, clinical significance, and treatment. J Am Soc Nephrol 2017;28:1023-39.

7 Tuttle KR, Bakris GL, Bilous RW, et al. Diabetic kidney disease: a report from an ADA consensus conference. Am J Kidney Dis 2014;64:510-33.

8 Zoungas S, de Galan BE, Ninomiya T, et al. Combined effects of routine blood pressure lowering and intensive glucose control on macrovascular and microvascular outcomes in patients with type 2 diabetes: new results from the advance trial. Diabetes Care 2009;32:2068-74.

9 Zatz R, Dunn BR, Meyer TW, et al. Prevention of diabetic glomerulopathy by pharmacological amelioration of glomerular capillary hypertension. J Clin Invest 1986;77:1925-30.

10 Lytvyn Y, Bjornstad P, Pun N, et al. New and old agents in the management of diabetic nephropathy. Curr Opin Nephrol Hypertens 2016;25:232-9.
11 Wanner C, Inzucchi SE, Lachin JM, et al. Empagliflozin and progression of kidney disease in type 2 diabetes. N Engl $\mathrm{J}$ Med 2016;375:323-34.

12 Neal B, Perkovic V, Mahaffey KW, et al. Canagliflozin and cardiovascular and renal events in type 2 diabetes. N Engl J Med 2017;377:644-57.

13 Marso SP, Bain SC, Consoli A, et al. Semaglutide and cardiovascular outcomes in patients with type 2 diabetes. N Engl $\mathrm{J} \mathrm{Med}$ 2016;375:1834-44.

14 Hoerger TJ, Wittenborn JS, Segel JE, et al. A health policy model of CKD: 2. The cost-effectiveness of microalbuminuria screening. Am J Kidney Dis 2010;55:463-73.

15 Komenda P, Ferguson TW, Macdonald K, et al. Cost-effectiveness of primary screening for CKD: a systematic review. Am J Kidney Dis 2014:63:789-97.

16 Stern E, Benbassat CA, Goldfracht M. Impact of a two-arm educational program for improving diabetes care in primary care centres. Int J Clin Pract 2005;59:1126-30.

17 Barlow J, Krassas G. Improving management of type 2 diabetes - findings of the Type2Care clinical audit. Aust Fam Physician 2013;42:57-60.

18 Horný M, Glover W, Gupte G, et al. Patient navigation to improve diabetes outpatient care at a safety-net hospital: a retrospective cohort study. BMC Health Serv Res 2017;17:759.

19 Umar-Kamara M, Tufts KA, Adams Tufts K. Impact of a quality improvement intervention on provider adherence to recommended standards of care for adults with type 2 diabetes mellitus. J Am Assoc Nurse Pract 2013;25:527-34.

20 Cleghorn GD, Nguyen M, Roberts B, et al. Practice-based interventions to improve health care for Latinos with diabetes. Ethn Dis 2004;14:S117-21.

21 Baty PJ, Viviano SK, Schiller MR, et al. A systematic approach to diabetes mellitus care in underserved populations: improving care of minority and homeless persons. Fam Med 2010;42:623-7.

22 Hughes-Carter DL, Hoebeke RE. Screening for diabetic kidney disease in primary care for the underinsured: a quality improvement initiative. App/ Nurs Res 2016;30:148-53.

23 Anabtawi A, Mathew LM. Improving compliance with screening of diabetic patients for microalbuminuria in primary care practice. ISRN Endocrinol 2013;2013:893913

24 Deem M, Rice J, Valentine K, et al. Screening for diabetic kidney disease in primary care: a quality improvement initiative. Nurse Pract 2020;45:34-41.

25 Choudhary D, Brown B, Khawar N, et al. Implementation of electronic medical record template improves screening for complications in children with type 1 diabetes mellitus. Pediatric Health Med Ther 2020;11:219-23.

26 Herrin J, da Graca B, Aponte P, et al. Impact of an EHR-based diabetes management form on quality and outcomes of diabetes care in primary care practices. Am J Med Qual 2015;30:14-22.

27 Pageler NM, Longhurst CA, Wood M, et al. Use of electronic medical record-enhanced checklist and electronic dashboard to decrease CLABSIs. Pediatrics 2014;133:e738-46.

28 Anaikatti P, Sheth SK, Canlas AM, et al. Electronic medical record platform enhancements during COVID-19 to support identify-isolateinform strategy for initial detection and management of patients. Emerg Med Australas 2021;33:164-7.

29 Sheen Y-J, Huang C-C, Huang S-C, et al. Implementation of an electronic DASHBOARD with a remote management system to improve glycemic management among hospitalized adults. Endocr Pract 2020:26:179-91.

30 Wu DTY, Deoghare S, Shan Z, et al. The potential role of dashboard use and navigation in reducing medical errors of an electronic health record system: a mixed-method simulation handoff study. Health Syst 2019;8:203-14.

31 Breton J, Witmer CM, Zhang Y. Utilization of an electronic medical Record-integrated Dashboard improves identification and treatment of anemia and iron deficiency in pediatric inflammatory bowel disease. Inflamm Bowel Dis 2020.

32 Embi PJ, Leonard AC. Evaluating alert fatigue over time to EHRbased clinical trial alerts: findings from a randomized controlled study. J Am Med Inform Assoc 2012;19:e145-8.

33 Alagiakrishnan K, Ballermann M, Rolfson D, et al. Utilization of computerized clinical decision support for potentially inappropriate medications. Clin Interv Aging 2019;14:753-62.

34 Cook J. Laboratory integration and consolidation in a regional health system. Lab Med 2017;48:e43-52. 